\title{
Perspective Piece \\ Dexamethasone and COVID-19: Strategies in Low- and Middle-Income Countries to Tackle Steroid-Related Strongyloides Hyperinfection
}

\author{
Mario J. Olivera* \\ Grupo de Parasitología, Instituto Nacional de Salud, Bogotá, Colombia
}

\begin{abstract}
COVID-19 can trigger a systemic inflammatory response that in some cases leads to severe lung involvement, multisystem dysfunction, and death. Dexamethasone therapy, because of its potent anti-inflammatory effects, has been proposed for the management of hospitalized patients with severe COVID-19. The subject of this article is to discuss potential strategies to tackle Strongyloides hyperinfection in hospitalized patients with COVID-19 receiving dexamethasone therapy in low- and middle-income countries. In this context, dexamethasone treatment has been found to be generally safe. However, its use in people coinfected with undetected Strongyloides stercoralis increases the risk for Strongyloides hyperinfection/dissemination a potentially fatal complication. Infection caused by S. stercoralis may remain asymptomatic or with mild symptoms in humans for several years. Early detection and specific treatment prevent a fatal evolution of this complication, but the challenge is to screen before corticosteroid therapy. In some cases, presumptive treatment may be justified. Ivermectin is the gold standard for treatment.
\end{abstract}

\section{INTRODUCTION}

The management of severe and critical patients with COVID-19 remains a challenge because of the high mortality. Although several drugs approved for other indications, as well as new investigational agents, are being evaluated for the treatment of COVID-19, there are currently no effective drugs to treat this patient population. ${ }^{1}$ There may be some possibilities in the future, but at this time, there are no drugs approved for the treatment of COVID-19. A recent clinical trial in the United Kingdom found that dexamethasone, a corticoid used in a variety of conditions for its anti-inflammatory and immunosuppressive effects, provides benefits to critically ill patients with COVID-19, leading to increased use of this therapy. $^{2}$

In general, dexamethasone treatment is safe, usually used in low doses and for short periods of time. However, its use in patients coinfected with undetected Strongyloides stercoralis can generate severe, rare complications such as hyperinfection, as well as dissemination with a wide migration of larvae that is often accompanied by severe enterocolitis and potentially fatal Gram-negative sepsis. ${ }^{3,4}$ This article aims to discuss potential strategies to tackle Strongyloides hyperinfection in hospitalized patients with COVID-19 receiving dexamethasone therapy in low- and middle-income countries.

In this context, it is important to consider strongyloidiasis when using immunosuppressive therapies for treatment with COVID-19. Clinical suspicion of hyperinfection and disseminated strongyloidiasis should be maintained in patients with immunosuppression or steroid treatment who develop opportunistic or atypical infection. Early detection is a challenge in this clinical setting, given the rapidity with which COVID-19 can progress to severe disease and because of the nonspecificity of the signs and symptoms of strongyloidiasis that lead to clinical..$^{3-5}$

The main difficulty in detecting S. stercoralis infections lies in the low sensitivity of the available diagnostic methods and in

*Address correspondence to Mario J Olivera, Grupo de Parasitología, Instituto Nacional de Salud, Ave. Calle 26 51-20, Bogotá DC, Colombia 111321. E-mail: molivera@ins.gov.co the biology of this nematode, where the adult female releases eggs and/or larvae intermittently, which makes it extremely difficult for its detection. Unlike hyperinfection syndromes, where diagnosis is relatively easy, chronic infections, usually asymptomatic, require strong clinical suspicion. . $^{6,7}$

In terms of diagnosis, there are different methods to detect the presence of $S$. stercoralis. Unfortunately, commonly used stool-based methods have low sensitivity. Examination of various stool samples, as well as concentration techniques, including the formol-ether technique, improves the performance of microscopy, which nevertheless remains insufficiently sensitive. Baermann's method and agar plate culture are preferable, although they are laborious and not used routinely. Other methods that have been developed include the ELISA, the indirect fluorescent serum antibody test, and the PCR. However, all these techniques have sensitivity, specificity, or availability problems in endemic areas. ${ }^{6-8}$

Among all the techniques, concentration techniques are frequently used in low- and middle-income countries, and by contrast, ELISAs, which seem to have the highest sensitivity and specificity, are used in high-income countries. However, serology may remain positive after resolution of the infection and the presence of cross-reactivity with other helminth infections, particularly filariasis, schistosomiasis and hookworm. ${ }^{9}$

In this scenario of uncertainty and with the intention of providing recommendations to clinicians on the detection and management of hyperinfection by Strongyloides among patients with COVID-19 who require the use of dexamethasone, some strategies have been proposed to address the complicated issue of serological diagnoses and that amid limited evidence, these are not helpful to physicians in low- and middle-income countries who are also endemic for the disease. ${ }^{4,10}$

In a recent study, an adequate and complete approach is made on this issue, and a test-and-treatment strategy for outpatients and for patients with mild COVID-19 using serological tests is suggested. For patients with COVID-19 who are, or may become, candidates for dexamethasone, it is suggested to consider presumptive treatment with ivermectin 
for moderate- to high-risk patients who have not been previously treated for Strongyloides and in cases where the serological tests are not available. This epidemiological stratification of the risk of infection to Strongyloides is given according to the country of birth or residence or long-term travel. ${ }^{4}$

Clearly, these recommendations can be of great use for high-income countries that are not endemic for the disease but have a migrant population. ${ }^{11}$ However, this approach could not be carried out in everyday practice in tropical lowand middle-income countries because serological tests for S. stercoralis are not available in the first place (a common scenario in these countries). Second, because of the considerable cross-reactivity with other nematode species that are also prevalent in these countries. This fact is particularly important given that the population has also been exposed to infection by other helminths for many years. And third, the difficulty of differentiating an old from a recent infection, which would require additional tests such as the Baermann method or PCR, which are not routinely available either. ${ }^{6-9}$

This diagnostic challenge has led many of the management protocols for COVID-19 in low- and middle-income countries to recommend a prophylactic dose of ivermectin for S. stercoralis before starting the corticosteroid. However, this prophylaxis may not be necessary in all cases. But what is relevant is knowing which individuals should be investigated for S. stercoralis infection before starting steroid therapy? And which diagnostic tests can we use in a scenario of unavailable serological tests?

To try to answer these questions in the contexts of routine practice in low- and middle-income countries, the following strategy is suggested:

Testing and treatment strategy for non-COVID-19 patients and for COVID-19 patients using formol-ether concentration techniques. It is the best fecal diagnostic method currently available for fieldwork. This method detects the parasite with greater sensitivity than the single stool examination. This test should be ordered in patients who have risk factors for $S$. stercoralis infection and in special groups. The history of poor housing conditions and poor sanitation, such as walking barefoot, living in areas where people usually defecate in the open, prisoners of war, and population displaced by violence, among others, will give greater certainty to whoever requests the test. ${ }^{12-14}$

For patients with confirmed diagnosis of COVID-19 who are, or may become, candidates for dexamethasone, it is suggested to consider a presumptive treatment with ivermectin for patients who could not undergo the formol-ether concentration techniques and who in addition to having factors of risk of infection has risk factors for dissemination such as a history of altered cellular immunity, human lymphotropic virus type 1 infection, neoplasms, particularly hematological neoplasms, and organ transplantation, among others.

A positive aspect of the pandemic is that it has extended diagnostic networks and improved access to molecular tests, interestingly loop-mediated isothermal amplification, with which it is expected that in the future, these diagnostic tools may be readily available, routinely. On the other hand, the serology for $S$. stercoralis is likely to be further improved by the use of recombinant antigens and is available in endemic areas. $^{9}$

Received August 26, 2020. Accepted for publication March 1, 2021.

Published online March 15, 2021.

Acknowledgment: Publication charges for this article were waived due to the ongoing pandemic of COVID-19.

Author's address: Mario J. Olivera, Grupo de Parasitología, Instituto Nacional de Salud, Bogotá, Colombia, E-mail: molivera@ins.gov.co.

This is an open-access article distributed under the terms of the Creative Commons Attribution (CC-BY) License, which permits unrestricted use, distribution, and reproduction in any medium, provided the original author and source are credited.

\section{REFERENCES}

1. McArthur L, Sakthivel D, Ataide R, Chan F, Richards JS, Narh CA, 2020. Review of burden, clinical definitions, and management of COVID-19 cases. Am J Trop Med Hyg 103: 625-638.

2. The RECOVERY Collaborative Group, 2020. Dexamethasone in hospitalized patients with Covid-19-preliminary report. NEngl J Med 384: 693-704

3. Mukaigawara M, Narita M, Shiiki S, Takayama Y, Takakura S, Kishaba T, 2020. Clinical characteristics of disseminated strongyloidiasis, Japan, 1975-2017. Emerg Infect Dis 26: 401-408.

4. Stauffer WM, Alpern JD, Walker PF, 2020. COVID-19 and dexamethasone a potential strategy to avoid steroid-related Strongyloides hyperinfection. JAMA 324: 623-624.

5. Lier AJ, Tuan JJ, Davis MW, Paulson N, McManus D, Campbell S, Peaper DR, Topal JE, 2020. Case report: disseminated strongyloidiasis in a patient with COVID-19. Am J Trop Med Hyg 103: 1590-1592.

6. Olivera MJ, Raciny M, López MC, Moncada L, Reyes P, 2014. Detection of Strongyloides stercoralis in Tierralta, Colombia using four parasitological methods. Rev Cuba Med Trop 66: 202-209.

7. Krolewiecki A, Nutman TB, 2019. Strongyloidiasis: a neglected tropical disease. Infect Dis Clin North Am 33: 135-151.

8. Requena-Méndez A, Chiodini P, Bisoffi Z, Buonfrate D, Gotuzzo E, Muñoz J, 2013. The laboratory diagnosis and follow up of strongyloidiasis: a systematic review. PLoS Negl Trop Dis 7: e2002.

9. Buonfrate D, Formenti F, Perandin F, Bisoffi Z, 2015. Novel approaches to the diagnosis of Strongyloides stercoralis infection. Clin Microbiol Infect 21: 543-552.

10. Olivera MJ, 2020. Opportunity cost and COVID-19: a perspective from health economics. Med J Islam Repub Iran 34: 34-177.

11. Requena-Méndez A, Buonfrate $D$, Gomez-Junyent J, Zammarchi L, Bisoffi Z, Muñoz J, 2017. Evidence-based guidelines for screening and management of strongloidiasis in non-endemic countries. Am J Trop Med Hyg 97: 645-652.

12. Schär F, Trostdorf $U$, Giardina $F$, Khieu V, Muth $S$, Marti $H$, Vounatsou P, Odermatt P, 2013. Strongyloides stercoralis: global distribution and risk factors. PLoS Negl Trop Dis 7: e2288.

13. Keiser PB, Nutman TB, 2004. Strongyloides stercoralis in the immunocompromised population. Clin Microbiol Rev 17: 208-217.

14. Manejo de la Estrongiloidiasis, 2018. World Gastroenterology Organisation Practice Guidelines. Available at: https://www. worldgastroenterology.org/UserFiles/file/guidelines/managementof-strongyloidiasis-spanish.pdf. Accessed January 11, 2021. 